\title{
Retrospective Surveillance for Intussusception in Children Aged Less than Five Years in a South Indian Tertiary-care Hospital
}

\author{
Kaushik Bhowmick', Gagandeep Kang',Anuradha Bose', Jacob Chacko', \\ Irving Boudville' ${ }^{2}$ Sanjoy K. Datta², and Hans L. Bock ${ }^{2}$ \\ 'Christian Medical College,Vellore 632 004, India and ${ }^{2}$ GlaxoSmithKline Biologicals, \\ Rue de l'Institut 89, 1330 Rixensart Belgium
}

\begin{abstract}
To facilitate the assessment of the safety profile of rotavirus vaccines effectively, baseline data on intussusception are important for comparison with intussusception rates following the introduction of vaccine. The aim of the study was to describe epidemiological and clinical features of intussusception in children aged less than five years in an Indian medical facility. Hospital data on intussusception for children discharged during 1 January 2001-30 June 2004 from the Christian Medical College Hospital, Vellore, India, were reviewed. Relevant information was extracted from medical records to classify cases according to the criteria of the Brighton Collaboration Intussusception Working Group. Complete review of medical records for clinical and demographic information was only performed for those cases fulfilling level 1 diagnostic certainty (definite intussusception) (Study ID 101245). During the surveillance period, 31 infants and children with definite intussusception were identified. The majority (61.2\%) of the cases occurred in the first year of life. The male : female ratio was $3.4: 1$. Intussusception cases occurred round the year with no distinct seasonality. No intussusception-associated death was recorded. This study provides baseline data on intussusception in South India. Cases identified in the study were similar in presentation and demographics as those observed in other Asian settings. Prospective surveillance systems, using standardized case definitions will further increase the understanding of the aetiology and epidemiology of intussusception, especially as new rotavirus vaccines are made available.
\end{abstract}

Key words: Diarrhoea, Infantile; Intussusception; Retrospective studies; Rotavirus; Rotavirus vaccines; India

\section{INTRODUCTION}

Intussusception is the most common cause of acute intestinal obstruction in infants and young children (1), with approximately two-thirds of all intussusceptions in children occurring among infants aged less than one year. Some cases of intussusception resolve spontaneously and, if treated early, most can be reduced by oenema or surgery, and if untreated, most have fatal outcomes (2). Anatomic conditions triggering intussusception

Correspondence and reprint requests should be addressed to:

Dr. Sanjoy K. Datta

Director, Clinical R\&D and

Medical Affairs-Biologicals South Asia

GlaxoSmithKline Biologicals

Rue de l'Institut 89

1330 Rixensart

Belgium

Email: Sanjoy.K.Datta@gsk.com are discovered in less than $15 \%$ of cases. Viruses, such as adenovirus and enterovirus, have been associated with some cases (3).

Mortality caused by intussusception in infants and children is now uncommon in developed countries due to better access to healthcare facilities. In contrast, intussusception-associated mortality remains high in some developing countries (1). A recent and extensive review by the World Health Organization (WHO) on intussusception concluded that, in developed countries, the baseline incidence of intussusception is between 0.5 and 4.3 cases per 1,000 livebirths or 0.7-1.2 cases per 1,000 children aged less than one year (4). Accurate estimates of the incidence of intussusception are not available for most developing countries (1).

In recent years, intussusception has attracted additional attention of the paediatric medical and public-health community because of its association 
observed in the United States with the first-licensed rotavirus vaccine-the rhesus-human reassortant rotavirus tetravalent (RRV-TV) vaccine $(5,6)$ - and the ensuing recommendations for its suspension nine months after its introduction (7). These events raised serious questions about the further development of other rotavirus candidates.

Rotavirus is the most common cause of severe diarrhoea among infants and young children (8), resulting in approximately 600,000 to 850,000 deaths annually worldwide (1). In India, rotavirusassociated acute gastroenteritis accounts for 5-70\% of all hospitalizations. About $20-30 \%$ of total hospitalizations are due to rotavirus-associated diarrhoea in early childhood (9). As a result of substantial morbidity and mortality worldwide caused by rotavirus, the WHO and Global Alliance for Vaccines and Immunization (GAVI) have identified rotavirus vaccines as a priority for development (10) and subsequent introduction (11).

Although clinical studies have demonstrated the next generation of rotavirus vaccines to be safe (12), baseline knowledge of the clinical presentation, treatment outcomes, and epidemiology of intussusception in infants and children in India, prior to the introduction of vaccine, is critically important because it will better inform interpretation of data on intussusception collected after these vaccines become available (4).

This study outlines the clinical presentation, detection, seasonality, management, and outcome of intussusception in young children aged less than five years in Vellore, India.

\section{MATERIALS AND METHODS}

This retrospective hospital-based study to review cases of intussusception was carried out at the Christian Medical College (CMC) Hospital, Vellore, India. The CMC, Vellore, occupies a prominent place among healthcare institutions in India. It is a 2,234-bed multispeciality, tertiary-care teaching hospital, which consists of a vital, diverse, and inter-denominational community (13). The protocol received appropriate institutional ethical review.

Surveillance to identify cases of intussusception was planned for three complete years. However, the investigators included four subjects beyond the defined surveillance period, and therefore, the study period was extended up to June 2004. During 1 January 2001-30 June 2004, cases of intussusception were identified among hospitalized children aged less than five years.
A case was identified in a two-step process: Possible cases of intussusception were first identified by searching radiology, pathology and computerized discharge-records of hospital for the diagnoses based on the International Classification of Diseases, Ninth and Tenth Revision, Clinical modification codes for intussusception (ICD-9 CM), and intestinal obstruction (ICD-10 CM). The diagnosis of intussusception was then confirmed by reviewing the medical records.

Once a potential case was identified, the study medical officer reviewed the medical records of the child. Cases of intussusception, which met the case definition with level 1 diagnostic certainty by the criteria (surgical/radiological/ autopsy) of the Brighton Collaboration Intussusception Working Group, were included in the study (6).

Abstracted data included demographic information, clinical manifestations, past medical history, including diagnostic procedures, treatment, and patient outcomes. The data were analyzed using the SAS software (version 8.2).

\section{RESULTS}

In total, 32 cases of intussusception in the agegroup of 0-5 year(s) were identified during the surveillance period; one case was excluded from analysis as the child was diagnosed with intussusception exclusively based on clinical signs and symptoms. Hence, 31 definite cases of intussusception were considered for analysis.

The median age of subjects was nine (range 1-55) months. The majority (61.2\%) of these cases were infants with a peak between 6 and 12 months of age (Fig. 1). The male : female ratio was $3.4: 1$. A male preponderance was observed ( 24 males, 7 females). All the subjects were Indians.

Intussusception cases occurred round the year with no distinct seasonality (Fig. 2). Vomiting (26 cases; $83.9 \%$ ), pain in the abdomen (23 cases; $74.2 \%$ ), abdominal mass (19 cases; 61.3\%), and red currant jelly stool (13 cases; $41.9 \%$ ) were the most frequently-recorded clinical signs and symptoms (Table 1). Of the 31 infants and children with intussusception, four (12.9\%) had a past history of gastrointestinal emergencies. Overall, four cases had received vaccination within the month preceding hospitalization: the first against diphtheriatetanus-pertussis (DTP), polio (OPV), Haemophilus influenzae (Hib), and hepatitis B (HBV); the second against DTP; the third against measles; and the 


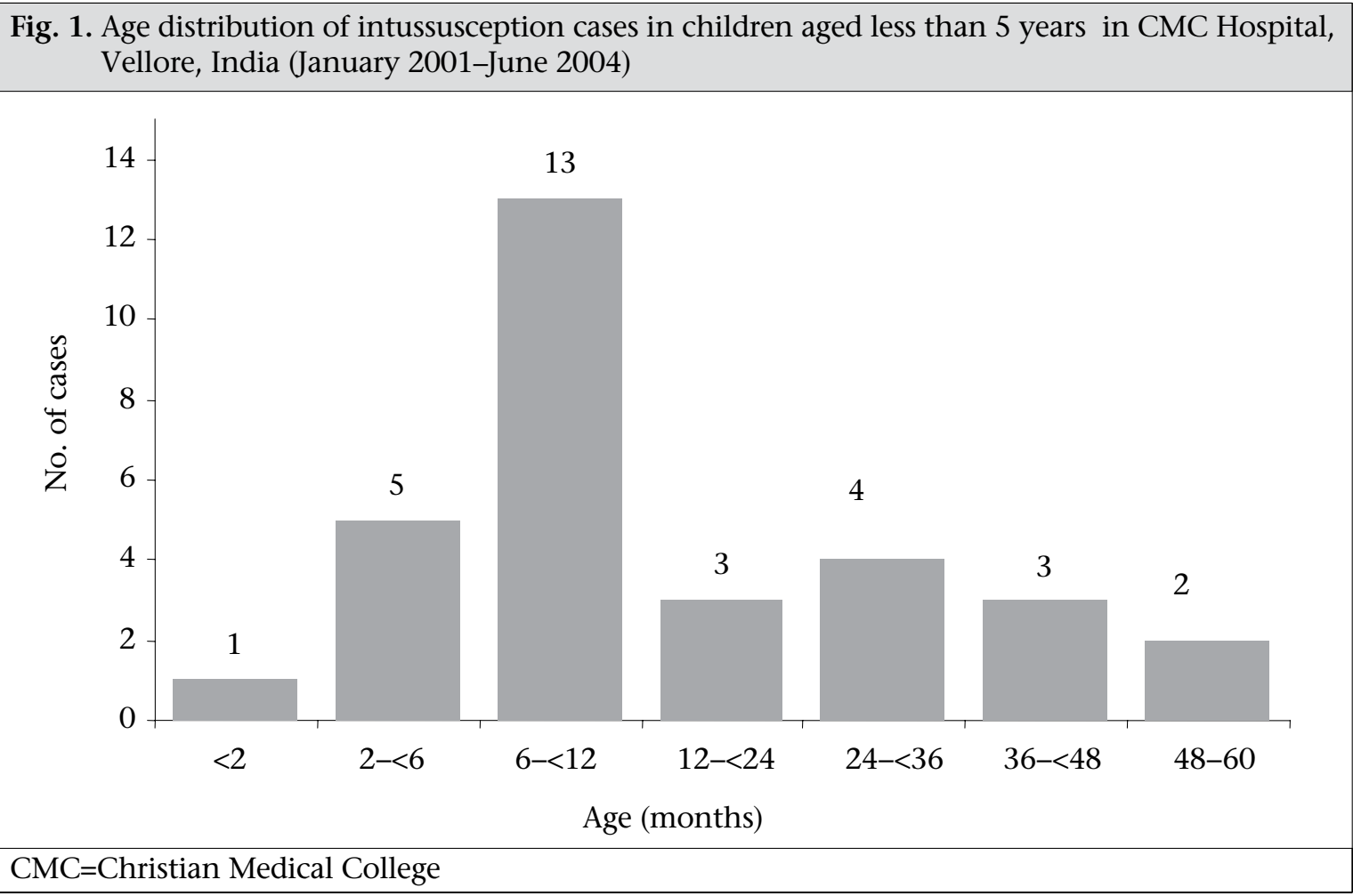

Fig. 2. Seasonality of intussusception cases in CMC, Hospital, Vellore, India, January 2001-December 2003*

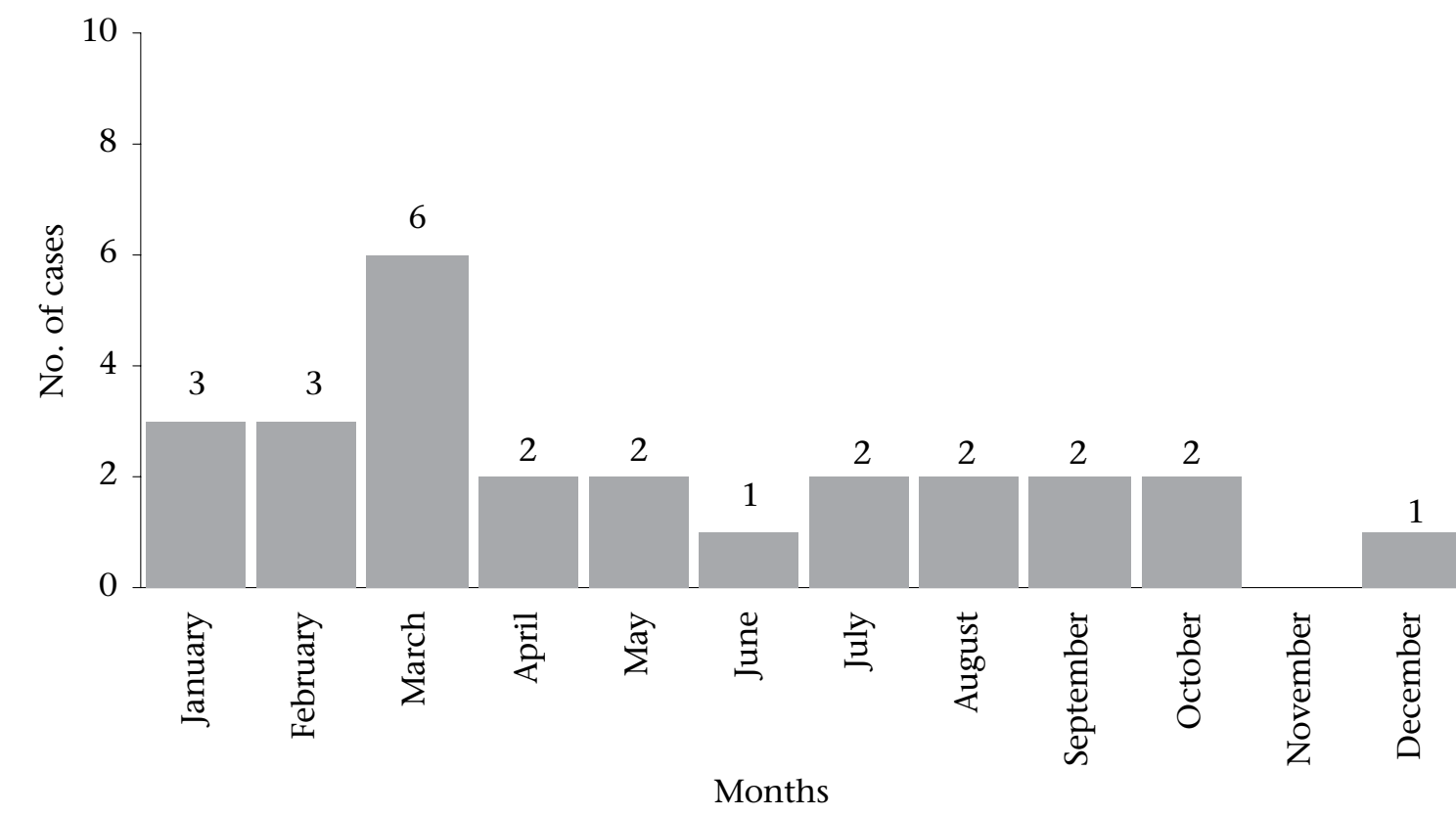

${ }^{*}$ Four cases reported during January-June 2004 (one each in January and March, two in May) were not included; $\mathrm{CMC}=$ Christian Medical College

fourth against DTP and OPV. However, for 22 cases, recent vaccination history was unknown. Thirtytwo percent (10/31) of the cases presented to the hospital for treatment beyond two days from the onset of symptoms (Table 2).
Less than half (41.9\%) of the intussusception cases were diagnosed by barium enema, although diagnosis following surgical procedure was still common (35.5\%). Other cases were diagnosed either by a combination of these two procedures (12.9\%) or barium enema com- 


\begin{tabular}{|lc|}
\hline $\begin{array}{c}\text { Table 1. Clinical signs and symptoms reported } \\
\text { among 31 intussusception cases in } \\
\text { CMC Hospital, Vellore, India (January } \\
\text { 2001-June 2004) }\end{array}$ \\
\hline Clinical feature & $\%$ \\
\hline Abdominal mass & 61.3 \\
Pain in abdomen & 74.2 \\
Diarrhoea & 19.4 \\
Fever & 22.6 \\
Red currant jelly stool & 41.9 \\
Vomiting & 83.9 \\
Other & 71.0 \\
\hline CMC=Christian Medical College \\
\hline
\end{tabular}

Table 2. Distribution of days between onset of symptoms of intussusception and admission to hospital

\begin{tabular}{|lcc|}
\hline $\begin{array}{l}\text { Days between onset } \\
\text { and admission }\end{array}$ & No. of cases & \% of cases \\
\hline 0 & 4 & 12.9 \\
1 & 9 & 29.0 \\
2 & 8 & 25.8 \\
3 & 4 & 12.9 \\
5 & 4 & 12.9 \\
7 & 1 & 3.2 \\
8 & 1 & 3.2 \\
\hline
\end{tabular}

bined with ultrasound (6.5\%). Diagnosis exclusively by ultrasound only occurred in $3.2 \%$ of the cases.

Surgical intervention was required in 20 cases (64.5\%), radiological reduction in 10 cases (32.3\%), and a combination of these two procedures in one case (3.2\%). The median duration of hospitalization for the intussusception cases in this study was 5.0 (range 1-13) days. One patient was admitted to the Intensive Care Unit. All intussusception cases resolved, and no death was recorded.

\section{DISCUSSION}

Our study retrospectively analyzed 3.5 years of intussusception data in the CMC Hospital, Vellore, India. Although there are a few private hospitals with modern facilities in the surrounding areas of Vellore, most middle- and lower-class children living in this area would have been seen at the CMC Hospital. In addition, the CMC Hospital is a tertiary referral centre in India and, therefore, receives paediatric referral cases from surrounding areas as well.

In many countries, incidence data in hospital-based studies are hard to obtain because denominator populations are not available to match numerator data, such as on hospitalizations. Similarly, in our study, the denominator of births was unavailable and would, in any case, have been subject to underascertainment owing to the high proportion of childbirths in the home. Furthermore, the catchment area for numerator cases of intussusception was difficult to define because of referrals to the CMC Hospital from other hospitals in Vellore and the surrounding towns. For these reasons, incidence was not estimated. Further research, including details of local healthcare-seeking behaviour, would be required to enable an estimate of population-based incidence rates of health events, such as intussusception.

Nonetheless, these data from a three-year period provide a reasonable baseline for occurrence of intussusception in this area. If we make the reasonable assumption that the catchment population served by the CMC Hospital will remain stable, this baseline will still be useful for the evaluation of intussusception trends in the future.

Meanwhile, this study usefully describes the epidemiological pattern of intussusception in Vellore. Male predominance, which is observed globally and in previous studies conducted in Asia (1), is reaffirmed in this study. No clear seasonality trend was observed in our study, which is consistent with reports from other studies $(14,15)$. In this study, the classic triad of abdominal pain, vomiting, and rectal bleeding was not the typical clinical manifestations in the intussusception cases. However, clinical signs and symptoms in our study population were similar to those reported previously in other studies (16). Vomiting was the most common clinical symptom. Its absence makes the diagnosis of intussusception unlikely. Over half of the subjects sought medical attention beyond two days after onset of the disease. The frequency of late presentation led to a relatively high rate of surgery, with over one-third of the cases being diagnosed only at surgery and almost two-thirds requiring surgical intervention.

The retrospective nature of this study does mean that it has some limitations. First, it is unclear how inter-hospital transfers and re-admissions for the same intussusception episode are identified to avoid duplication of cases (17). Second, our study was limited by the lack of complete immunization data which made it difficult to reliably count the number of immunizations given prior to hospi- 
talization for intussusception. This was, however, not a main objective of the study. Finally, our study was limited by the inability to define the catchment area for intussusception cases or to obtain accurate birth-cohort data for the catchment population. Nevertheless, the strength of this retrospective study is that it analyzed data from a tertiary hospital with complete investigation and reliable documentation, and cases of intussusception were ascertained using a standard case definition. The specificity of case ascertainment is, therefore, likely to be high, and the description of clinical presentation, treatment outcomes, and epidemiology is consequently an accurate representation of intussusception in the population in Vellore.

The study provides important insights into the epidemiology of intussusception among southern Indian children. Although the next generation of licensed rotavirus vaccines show no association with intussusception $(18,19)$, this does not negate the need for post-marketing surveillance which would provide re-assuring further confirmation of their safety-this study provides baseline data to facilitate further surveillance in this area.

\section{ACKNOWLEDGEMENTS}

The authors thank the doctors who contributed to the study and Clinical Research Associate, Namitha Krishnamurthy, for local monitoring support. They also thank Priya Rajagopal for her support in statistical analysis, Manjula K and Avishek Pal for medical writing, Veronique Delpire for editorial assistance, and Melvin D' Paul and Ming Tung Lim for publication coordination.

GlaxoSmithKline Biologicals was the funding source and was involved in all stages of conduct of the study and analysis. GlaxoSmithKline Biologicals paid all costs associated with the development and publication of the paper. The corresponding author had full access to the data and had the final responsibility for submission of the publication.

\section{REFERENCES}

1. WHO vaccines and biologicals. Acute intussusception in infants and children. Incidence, clinical presentation and management: a global perspective. Geneva: World Health Organization, 2002:1-98.

2. Parashar UD, Holman RC, Cummings KC, Staggs NW, Curns AT, Zimmerman CM et al. Trends in intussusception-associated hospitalizations and deaths among US infants. Pediatrics 2000;106:1413-21.

3. O’Ryan M, Lucero Y, Pena A, Valenzuela MT. Two year review of intestinal intussusception in six large public hospitals of Santiago, Chile. Pediatr Infect Dis J 2003;22:717-21.

4. Nakagomi T, Takahashi Y, Arisawa K, Nakagomi O. A high incidence of intussusception in Japan as studied in a sentinel hospital over a 25-year period (19782002). Epidemiol Infect 2006;134:57-61.

5. Glass RI, Bresee JS, Parashar UD, Jiang B, Gentsch J. The future of rotavirus vaccines: A major setback leads to new opportunities. Lancet 2004;363:1547-50.

6. Bines JE, Kohl KS, Forster J, Zanardi LR, Davis RL, Hansen $\mathrm{J}$ et al.; Brighton Collaboration Intussusception Working Group. Acute intussusception in infants and children as an adverse event following immunization: case definition and guidelines of data collection, analysis, and presentation. Vaccine 2004;22:569-574.

7. Centers for Disease Control and Prevention. Intussusception among recipients of rotavirus vaccineUnited States, 1998-1999. MMWR 1999;48:577-81.

8. Banerjee I, Ramani S, Primrose B, Moses P, IturrizaGomara M, Gray JJ et al. Comparative study of the epidemiology of rotavirus in children from a community-based birth cohort and a hospital in South India. J Clin Microbiol 2006;44:2468-74.

9. Phukan AC, Patgiri DK, Mahanta J. Rotavirus associated acute diarrhoea in hospitalized children in Dibrugarh, north-east India. Indian J Pathol Microbiol 2003;46:274-8.

10. Glass RI, Bresee JS, Turcios R, Fischer TK, Parashar UD, Steele AD. Rotavirus vaccines: targeting the developing world. J Infect Dis 2005;92 (Suppl 1):S160-6.

11. Global initiative to fast-track vaccine for world's leading cause of severe diarrhea among children. Project expected to speed rotavirus vaccine availability to developing countries by $15-20$ years. (http://www. globalhealth.org/news/article/2805, accessed on 8 December 2006).

12. Glass RI, Parashar UD. The promise of new rotavirus vaccines. N Engl J Med 2006;354:75-7.

13. Christian Medical College Hospital, Vellore. (http:// home.cmcvellore.ac.in, accessed on 8 December 2006)

14. Boudville IC, Phua KB, Quak SH, Lee BW, Han HH, Verstraeten $\mathrm{T}$ et al. The epidemiology of paediatric intussusception in Singapore: 1997 to 2004. Ann Acad Med Singapore 2006;35:674-9.

15. Rennels MB, Parashar UD, Holman RC, Le CT, Chang HG, Glass RI. Lack of an apparent association between intussusception and wild or vaccine rotavirus infection. Pediatr Infect Dis J 1998;17:924-5. 
16. Eshel G, Barr J, Heyman E, Tauber T, Klin B, Vinograd I et al. Intussusception: a 9-year survey (1986-1995). J Pediatr Gastroenterol Nutr 1997;24:253-6.

17. Grimwood K. Unraveling rotaviruses, oral vaccines and intussusception. J Paediatr Child Health 2005;41:471-2.

18. Linhares AC, Velázquez FR, Pérez-Schael I, SáezLlorens X, Abate H, Espinoza F et al. Efficacy and safety of an oral live attenuated human rotavirus vaccine against rotavirus gastroenteritis during the first 2 years of life in Latin American infants: a randomised, double-blind, placebo-controlled phase III study. Lancet 2008;371:1181-9.

19. Vesikari T, Matson DO, Dennehy P, Damme PV, Santosham M, Rodriguez $\mathrm{Z}$ et al. Safety and efficacy of a pentavalent human-bovine (WC3) reassortant rotavirus vaccine. N Engl J Med 2006;354:23-33. 